\title{
EL TURISMO COMO UNA HERRAMIENTA PARA LA CONSERVACIÓN DEL ELEFANTE MARINO DEL SUR (MIROUNGA LEONINA) Y SUS HABITATS EN TIERRA DEL FUEGO, RESERVA DE LA BIOSFERA CABO DE HORNOS, CHILE
}

\author{
CHRISTIAN KIRK ${ }^{\mathrm{a}}$, RICARDO ROZZI ${ }^{\mathrm{b}}$, STEFAN GELCICH ${ }^{\mathrm{c}}$
}

\begin{abstract}
RESUMEN
El elefante marino del sur (Mirounga leonina Linnaeus 1758) es una especie poco común en las costas chilenas fuera del territorio antártico chileno. En Tierra del Fuego se han descrito colonias estables y reproductivas de esta especie, que se ha identificado como el primer mamífero marino en ser utilizado turísticamente en la región de Magallanes y Antártica Chilena. Con el fin de establecer la importancia de la conservación de esta especie y sus hábitats para los turistas, se realizaron 355 encuestas a pasajeros de cruceros que visitan el seno Almirantazgo y otras zonas de la Cordillera Darwin en la Reserva de la Biosfera Cabo de Hornos. Mediante encuestas se evaluó el perfil de turistas que realizan la visita a esta reserva con la compañía Cruceros Australis, considerando su conocimiento sobre conservación del medioambiente y su voluntad de colaborar en un programa para la conservación del elefante marino y la biodiversidad y hábitats en la Patagonia chilena. Más del $76 \%$ de los encuestados mostraron una disposición a aportar a un programa de conservación de esta especie y a la biodiversidad y hábitats de la Patagonia. El 53\% de los encuestados estuvo dispuesto a contribuir con US $\$ 100$ o 200. Considerando que desde el año 2012 el número de turistas que se han embarcado anualmente en Cruceros Australis ha superado las 14 mil personas, su aporte total por año podría mayor que US $\$ 1.000 .000$. Este resultado que identifica una contribución potencialmente alta por parte de un número de turistas con intereses especiales es coherente con lo registrado para otras regiones del mundo, donde un área silvestre protegida bien conservada, como la Reserva de Biosfera Cabo de Hornos, posee un potencial para generar mayores aportes con menor número de visitantes que aquellas áreas protegidas con problemas de conservación y una visitación masiva, como ha ocurrido en la Reserva de la Biosfera Torres del Paine durante las últimas dos décadas.
\end{abstract}

PALABRAS CLAVE: áreas protegidas, biodiversidad, disposición a pagar, mamíferos marinos, turismo sustentable.

a Fundación Cordillera Darwin, Chile. $\$ christian@cordilleradarwin.org

b Programa de Conservación Biocultural SubAntártica, Universidad de Magallanes e Instituto de Ecología y Biodiversidad de Chile \& Department of Philosophy and Religion, University of North Texas, Denton, Texas USA. ricardo.rozzi@unt.edu

c Centro de Ecología Aplicada y Sustentabilidad (CAPES), P. Universidad Católica de Chile, Santiago, Chile. Fondo basal 0002.sgelcich@bio.puc.cl 


\section{TOURISM AS A TOOL FOR THE CONSERVATION OF THE SOUTHERN ELEPHANT SEAL (MIROUNGA LEONINA) AND ITS HABITATS IN TIERRA DEL FUEGO, CAPE HORN BIOSPHERE RESERVE, CHILE}

\section{ABSTRACT}

The southern elephant seal (Mirounga leonina Linnaeus 1758) is a rare species on the Chilean coast outside the Chilean Antarctic territory. In Tierra del Fuego, stable and reproductive colonies of this species have been identified, and it has been described as the first marine mammal to be used in the tourism industry in the Magellanic and Antarctic Region of Chile In order to establish the importance that the conservation of this species and its habitats has for tourists, 355 surveys were carried out on cruise ship passengers that visiting the Almirantazgo sound and other areas of the Darwin Cordillera in the Cape Horn Biosphere Reserve. Surveys assessed the profile of tourists who visit this reserve with the company Cruceros Australis, considering their knowledge about nature conservation and their willingness to collaborate in a program for the conservation of the elephant seal, that habitats and biodiversity of the Chilean Patagonia. More than $76 \%$ of the respondents showed a willingness to contribute to a conservation program of this species and the biodiversity of Patagonia. Among the respondents, 53\% were willing to contribute US $\$ 100$ or 200. Considering a number of 14 thousand tourists who embarked on Crucerso Australis in 2012, the total contribution per year could be greater than US $\$ 1,000,000$. This result that identifies a high potential contribution by tourists is consistent with the records for other regions of the world, where a well-preserved protected area (such as the Cape Horn Biosphere Reserve) has the potential to generate greater contributions with lower number of tourists than a protected area with massive numbers of tourists and conservation problems, as it has been the case of the Torres del Paine Biosphere Reserve during the last two decades.

KEY WORDS: biodiversity, marine mammals, protected areas, sustainable tourism, willingness to pay.

\section{INTRODUCCIÓN}

Las visitas no guiadas y sin control de turistas y sus actividades tienen un impacto negativo sobre las poblaciones de mamíferos marinos, generando cambios en sus sitios de alimentación o crianza, entre otros (Constantine, 1999). Por esta razón, resulta clave que los "destinos turísticos" tengan planes de manejo del número de visitantes y la forma de visitación. Es fundamental que las empresas de turismo tengan un alto compromiso y responsabilidad de conservación de las especies con el fin de permitir un desarrollo sustentable del turismo basado en la vida marina.

En el extremo sur de Chile, específicamente en Tierra del Fuego, se ha reportado por más de 30 años concentración de mamíferos marinos, especialmente en seno Almirantazgo, bahía Ainsworth, donde es posible avistar delfines (Lagenorinchus australis y Cephalorhynchus commersonii), lobos marinos (Arctocephalus australis y Otaria flavescens), elefante marino (Mirounga leonina, también llamado elefante marino del sur) y avistamientos ocasionales de orcas (Orcinus orca) y foca leopardo (Hydrurga leptonyx) durante las visitas turísticas (Rozzi et al. 2006a). Este sector se encuentra dentro de la Reserva de la Biosfera Cabo de Hornos (RBCH) que incluye los parques nacionales Alberto de Agostini, Cabo de Hornos (Rozzi et al. 2004), y más recientemente Yendegaia, que en sus aguas interiores posee la mayor concentración de mamíferos marinos del sur en la Región de Magallanes y Antártica Chilena (Gibbons, 2002). La RBCH se encuentra dentro de la ecorregión subantártica de Magallanes, una de las zonas marino-terrestres mejor conservadas del planeta y que posee atributos únicos respecto a su biodiversidad, calidad de las aguas y potencial para un turismo sustentable (Rozzi et al. 2012). 
Turismo sustentable y características del turismo regional

El turismo en la región de Magallanes representa una de las principales actividades económicas, alcanzando una participación del $7,1 \%$ en el PIB Regional y tasas de crecimiento promedio de $7 \%$ anual (SERNATUR, 2004). Actualmente, concentra más del $5,5 \%$ de la fuerza laboral de la región; esto es casi el doble que en otras regiones del país. Respecto a la llegada de turistas al área de la $\mathrm{RBCH}$, desde febrero de 1990 se iniciaron las operaciones de la empresa Cruceros Australis en la zona que han incluido la visita a la colonia de elefantes marinos de la bahía Ainsworth (Branco Ivelic comunicación personal, Fig. 1). En este sentido, Cruceros Australis ha sido la mayor empresa en términos de número de desembarcos en la Reserva de la Biosfera Cabo de Hornos durante los últimos 20 años. Las estadísticas de la empresa muestran que desde el año 2000 cerca de 4000 pasajeros anuales visitan bahía Ainsworth, y a partir del año 2012 el número superó los 14.000 pasajeros anuales. Es decir, se ha registrado un aumento de más de tres veces en poco más de 10 años de operaciones. La carga de visitantes ha sido mayor, ya que además han existido otros emprendimientos de embarcaciones menores que han realizado visitas esporádicas en el tiempo, entre ellas Antartic Dreams, Chonos, Nueva Galicia y actualmente Forrest (Sustentable, 2006; Cáceres, 2013).

Algunos estudios se refieren a los efectos del turismo sobre el elefante marino Mirounga leonina (Cáceres, 2013), no obstante no señalan la necesidad de mantener un equilibrio entre el desarrollo de la actividad turística y la conservación de esta especie. En otros países se ha demostrado que mayores números de visitantes en zonas naturales sin resguardo no sólo ponen en riesgo el valor de conservación de dichos lugares, sino también el sitio tiende a valorizarse cada vez menos. Esta desvalorización implica una menor disposición de los turistas a contribuir a su conservación, puesto que tales sitios pierden su valor diferenciador de ser únicos (Fig. 2) (Higginbottom, 2004; Higham \& Lück, 2007; Viteri \& Brandt, 2015). Esta proyección podría aplicarse a bahía Ainsworth y a su colonia de elefantes marinos del sur.

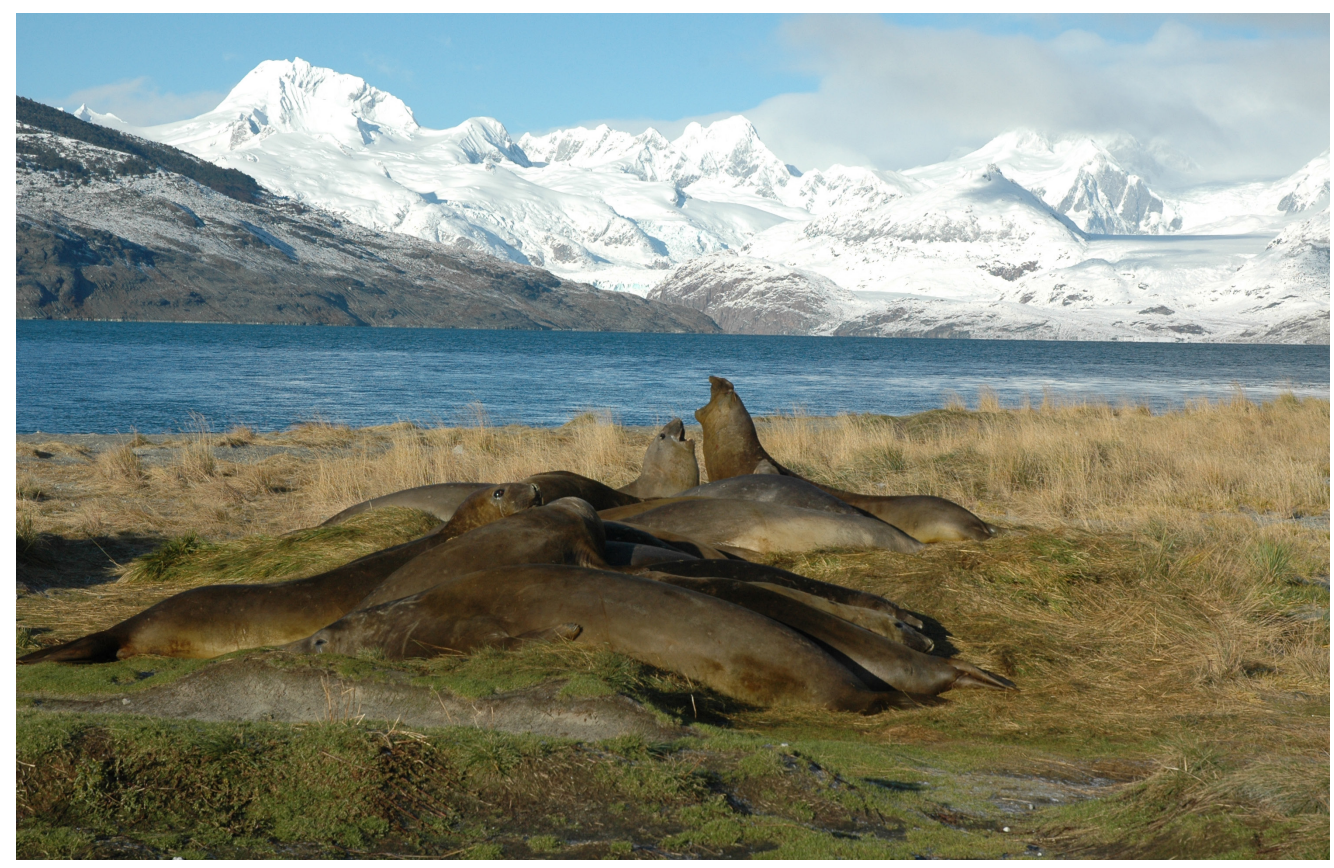

Fig. 1. Grupo de elefantes marinos (Mirounga leonina), también llamado elefante marino del sur, en bahía Anisworth, Seno Almirantazgo, Reserva de la Biosfera Cabo de Hornos. Al fondo se observa el glaciar Marinelli. Foto Ricardo Rozzi, Diciembre 2009. 
Posibles conflictos entre el turismo de naturaleza y la conservación

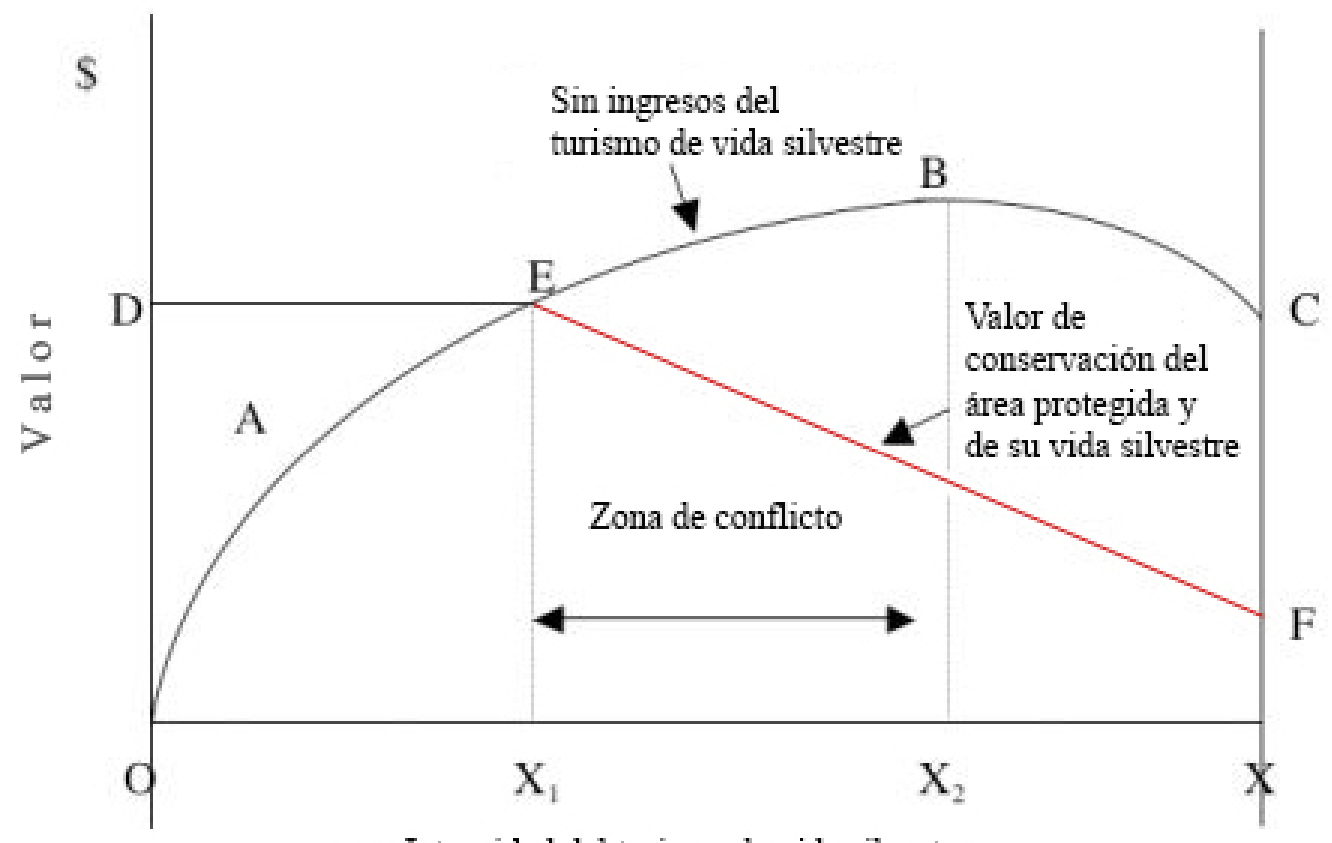

Intensidad del turismo de vida silvestre

Fig. 2. Modelo que demuestra el decaimiento del valor de un sitio a medida que aumenta el número de visitantes por sobre el valor "E". La curva OABC representa los ingresos netos que se pueden obtener en un área de vida silvestre protegida a partir del turismo de naturaleza. La línea DEF representa el valor de conservación de esta área y su vida silvestre. En este modelo el área protegida puede ser utilizada sin comprometer su valor de conservación hasta un número de visitantes X1. Hasta el número de turistas = X1, el turismo de naturaleza y la conservación son compatibles. Sin embargo, un número de turistas mayor que X1 comienzan a comprometer crecientemente el valor de conservación del área y su vida silvestre. Un número de turistas superior a X2 comienzan a disminuir los ingresos económicos derivados del turismo como también el valor de conservación del área y la vida silvestre. Figura adaptada de Higginbotton (2004, p. 153).

Especie de estudio

El elefante marino del sur (Mirounga leonina) es una de las cinco especies de fócidos del Hemisferio Sur. Los fócidos son una familia del orden Pinnipedia, al que también pertenecen las focas y las morsas. Entre las 18 especies de fócidos vivientes en el mundo, dos son elefantes marinos. Una de ellas, el elefante marino del norte, Mirounga angustirostris, tiene una distribución limitada al Pacífico norte. La especie del sur también pertenece al género Mirounga y hoy habita en una serie de islas subantárticas y en la península Antártica (Lewis, 1996). Para las costas chilenas no se han registrado grandes números poblacionales de esta especie en comparación con los que se encuentran en la actualidad en otras zonas subantárticas y en península Valdés. Sin embargo, en la región de
Magallanes se ha establecido una colonia con un número importante de elefantes marinos del sur en la porción chilena de la isla grande de Tierra del Fuego. Específicamente en bahía Ainsworth, en el seno Almirantazgo, en la $\mathrm{RBCH}$ se han registrado avistamientos por más de 30 años, por lo que podría considerarse una colonia reproductiva pequeña en territorio chileno (Gibbons \& Miranda, 2001; Lewis et al. 2006; Acevedo et al. 2016). Esta colonia posee alrededor de 20 individuos (Kirk observación personal) y tendría sus orígenes en ejemplares provenientes de la península Valdés y/o de las islas Malvinas (Acuña et al. 2005, Acevedo et al. 2016). Cabe destacar que en los últimos años se ha establecido otro grupo de elefantes marinos en el sector de caleta María, en el extremo este del seno Almirantazgo, en bahía Jackson. Este grupo alcanza ya un número cercano a los 60 
individuos (Kirk, obs. pers.; Acevedo et al. 2016). Finalmente, también se han registrado algunos grupos de elefantes marinos del sur en el sector norte de Puerto Natales (Gibbons, com. pers.).

Respecto a su estado de conservación, es esencial considerar que el elefante marino del sur fue sobreexplotado por su grasa como fuente de aceite en los siglos XIX y XX. Ello redujo su número considerablemente y la caza controlada de los machos adultos continuó en las islas Georgias del Sur hasta 1964 (Le Boeuf \& Laws, 1994). Actualmente, cualquier captura de esta especie al sur de los $60^{\circ} \mathrm{S}$ es regulada por la Convención para la Conservación de las Focas Antárticas de 1972. Más al norte, esta especie también está protegida por la legislación en varios países dentro de sus territorios. Debido a su presencia generalizada y al gran tamaño de la población a nivel mundial, el elefante marino del sur es clasificado como especie de Preocupación Menor por la Unión Internacional de Conservación de la Naturaleza (UICN). Sin embargo, la UICN advierte que debido a las recientes caídas poblacionales en algunas zonas, se deberían utilizar categorías de conservación a una escala que considere el estado de cada una de las poblaciones. En Chile, entre las especies avistadas en la $\mathrm{RBCH}$ el elefante marino se clasifica como "Insuficientemente Conocida" (MMA, 2013), y se encuentra sujeta a veda por 30 años de acuerdo al Decreto No 225 (1995) del Ministerio de Economía con el objeto de adoptar medidas de conservación que la protejan (CONAMA, 2002). El criterio de categoría de conservación según la población cobra relevancia en zonas naturales con poblaciones aisladas de elefante marino, especialmente cuando la carga de turistas es elevada y carece de regulación apropiada (UICN, 2014), como ocurre en el caso con la colonia de esta especie en la bahía Ainsworth en el sector sur de Tierra del Fuego (Kirk, 2012).

Hoy la colonia de elefantes marinos de bahía Ainsworth no cuenta con plan de manejo de visitas ni guardaparques, ni tampoco con estudios de capacidad de carga de turistas. La única información disponible por parte de CONAF es un informe de catastro de especies de 1982 (Aonken Consultores, 1982). En consecuencia, los criterios de conservación en las visitas a la colonia dependen hoy de la buena voluntad de la empresa que usa turísticamente el sector para acceder con el menor impacto posible. La colonia de elefantes marinos de la bahía Ainsworth constituye el primer caso de un mamífero marino utilizado turísticamente en la región de Magallanes y Antártica Chilena: desde febrero de 1990 se han realizado visitas periódicas a este sitio por la empresa Cruceros Australis (Branco Ivelic, com. pers.), teniendo como principal foco a esta especie. Las visitas se han realizado sin la presencia de guardaparques en la zona donde se encuentra la colonia de M. leonina, y tampoco se han elaborado planes de manejo de visitantes que cuenten con protocolos de avistamiento o acciones específicas de conservación (Kirk, 2012). El número anual de visitantes a esta colonia de elefantes marinos ha ido en aumento significativo durante los últimos años. A partir del año 2007 las visitas fueron del orden de 8000 visitantes anuales y a partir del 2012 las visitas superaron 14.000 turistas debido a que la empresa incorporó una nueva embarcación (Branco Ivelic comunicación personal), siempre concentrándose en el periodo comprendido entre los meses de octubre y abril.

Por otro lado, existe el potencial para que el turismo represente una oportunidad para la conservación de esta especie y de su hábitat. Esta actividad turística podría financiar y desarrollar programas de protección de la biodiversidad de la Patagonia y en especial del elefante marino, estableciendo las condiciones básicas para el avistamiento de la colonia que resguarden la dinámica poblacional de la especie y que de esa manera genere un equilibrio entre la conservación y el desarrollo sustentable de la actividad turística. En este contexto, este trabajo tiene por objeto evaluar el potencial que tiene el turismo en la bahía Ainsworth (Tierra del Fuego, Chile) para financiar un turismo sustentable en este sector $y$ en la Reserva de la Biosfera Cabo de Hornos en la Región de Magallanes y Antártica Chilena.

\section{MÉTODOS}

\section{Área de estudio}

El lugar que ocupa una colonia reproductiva de elefante marino del sur en Chile es la bahía Ainsworth (CONAMA, 2002; Gibbons \& Miranda, 2001; Lewis et al. 2006). Esta bahía recibe al glaciar Marinelli y se proyecta desde el 
seno Almirantazgo hacia el sur, en la extensión de Tierra del Fuego que alberga la cordillera Darwin. Se ubica dentro del Parque Nacional Alberto de Agostini en el sector norte de la $\mathrm{RBCH}$ (Fig. 3).

El cordón montañoso que se proyecta desde el este hacia al oeste en el sur de Tierra de Fuego se denomina Cordillera Darwin, en referencia al insigne explorador y científico británico Charles Darwin. Se ubica en los $55^{\circ}$ de latitud sur y presenta una alta pluviometría y una marcada disminución de las temperaturas en las altas cumbres que sobrepasan los $2000 \mathrm{~m}$ determinan la presencia de grandes glaciares (Rozzi et al. 2006a).

\section{Toma de datos}

En una primera etapa del trabajo se realizó una revisión de la bibliografía de Mirounga leonina en cuanto a características de su fisiología, ecología poblacional y ciclo de vida, y a las características de su hábitat en la $\mathrm{RBCH}$ con foco en el seno Almirantazgo, en especial la bahía Ainsworth. En una segunda etapa se realizaron encuestas presenciales a los turistas de las embarcaciones de la empresa Cruceros Australis. La primera parte del cuestionario incluyó preguntas tendientes a identificar el perfil del visitante, edad, cómo viaja, cómo se enteró del destino y otros aspectos en relación con la decisión de visitar el área. La segunda parte del cuestionario estaba relacionada con conocimiento y valoración que el turista le asigna a la conservación de la biodiversidad y del elefante marino en particular. La tercera sección del cuestionario se focalizó en la evaluación de la disposición a colaborar por parte del visitante en favor de la conservación del elefante marino en particular y de la biodiversidad en general de la región. El cuestionario incluyó preguntas estructuradas, semi-estructuradas y no-estructuradas o abiertas. Estas últimas permitieron responder al encuestado lo que deseara, en cambio, las preguntas estructuradas conllevaron una gama de opciones predeterminadas. El total de turistas que se embarcó en Cruceros Australis durante el período de cinco temporadas (20042008) alcanzó 23.461. Durante la temporada del año 2008 se encuestaron 355 visitantes,

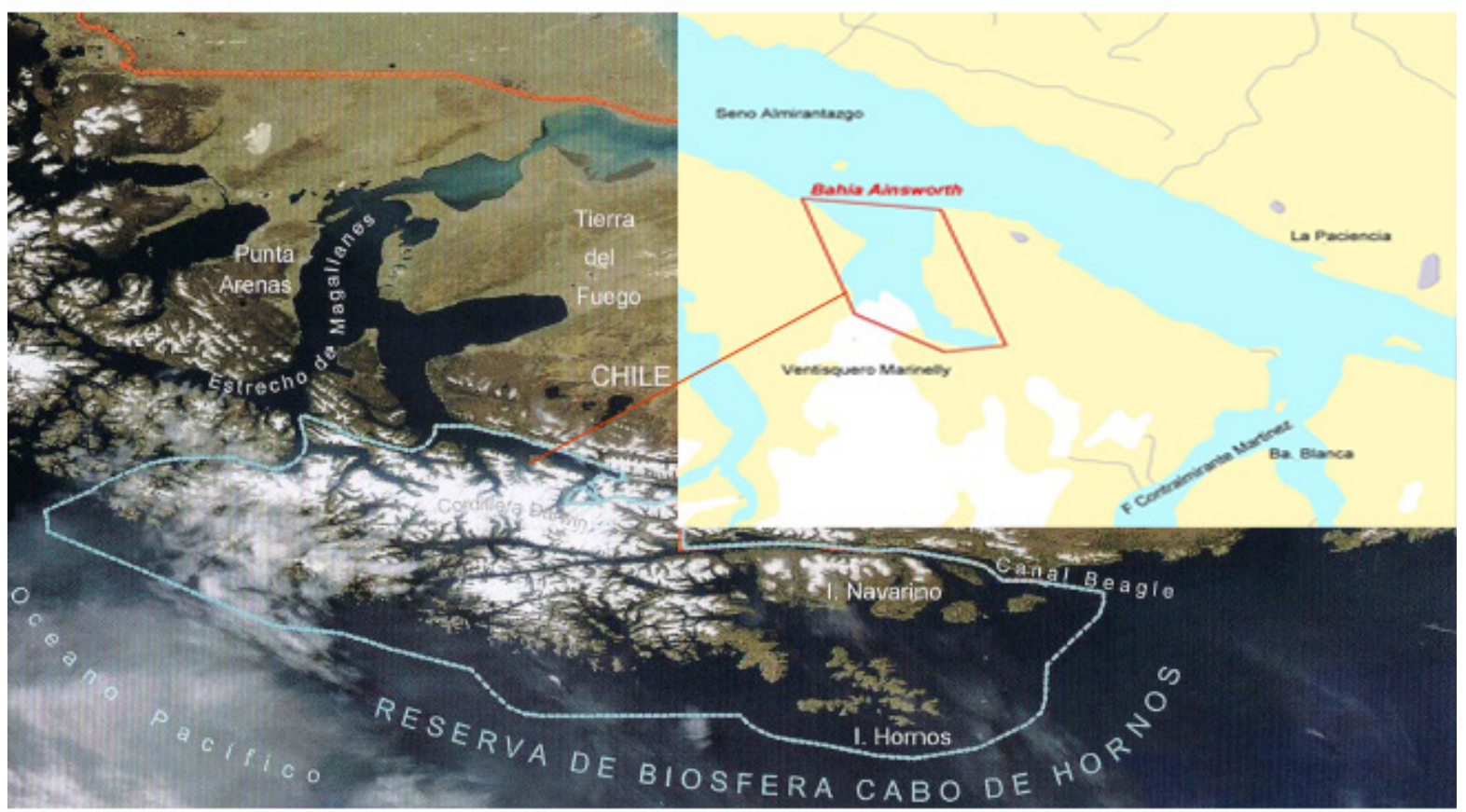

Fig. 3. Imagen satelital de la $\mathrm{RBCH}$, lugar en el cual la empresa Cruceros Australis realiza sus navegaciones turísticas. El inserto muestra el detalle de bahía Ainsworth donde se encuentra la colonia reproductiva de elefantes marinos en Tierra del Fuego. La línea en celeste señala los límites de la RBCH. Figura modificada a partir de Rozzi et al. (2006b). 
contando con la colaboración de guías turísticos dependientes de la empresa Cruceros Australis, quienes fueron capacitados para la recolección de los datos.

\section{Análisis de datos}

Una vez concluidas las etapas de obtención y procesamiento de los datos, a los resultados numéricos se les aplicó una prueba de normalidad. Dado que las respuestas al cuestionario no presentaron una distribución normal, se optó por utilizar pruebas estadísticas no paramétricas. Se utilizó la prueba de KruskalWallis para evaluar diferencias estadísticamente significativas entre las variables examinadas en esta investigación (Sokal \& Rohlf, 2012). Las diferencias significativas se evaluaron considerando una probabilidad de rechazo igual al 0,05. Cuando se encontró alguna significación entre los grupos, se aplicó una prueba de Dunn para comparaciones múltiples. Todos los análisis estadísticos se realizaron usando el programa estadístico SPSS versión 11.5 (SPSS).

\section{RESULTADOS}

Los resultados se describen en etapas: (1) descripción del perfil del turista, (2) conservación del elefante marino y (3) disposición a pagar para la conservación del elefante marino y los hábitats de la $\mathrm{RBCH}$. La medida de la dispersión usada en las preguntas 9 a la 14 corresponde a la desviación estándar.

\section{Descripción del perfil del turista}

Del total de los encuestados, los hombres representaron un $59 \%$ y las mujeres un $41 \%$. Respecto a la nacionalidad de los turistas, el mayor porcentaje fue de EE.UU. $(46,2 \%)$, seguido por España $(9,6 \%)$, Chile $(7,0 \%)$, Inglaterra $(5,9 \%)$, Italia $(4,5 \%)$ y Brasil (4,5\%). En términos del tiempo destinado exclusivamente para visitar la Patagonia, se estimó que los turistas permanecen entre los 5 a 15 días, con un promedio de 11 días. Respecto a las preferencias del tipo de turismo, los encuestados se inclinaron por el turismo de naturaleza, el ecoturismo y el turismo rural. Le siguieron el turismo de relax, deporte aventura, de sol y playa, y de salud. El menos valorado fue el turismo de negocios. Respecto a las motivaciones que determinan la realización de su viaje, las principales preferencias apuntan a vivir nuevas experiencias, la naturaleza, la tranquilidad, la relajación y a tener tiempo para la familia. Luego, siguieron el deporte y la salud.

Respecto a los factores más importantes para decidir y planificar su viaje, la mayoría de los visitantes valoró la belleza natural (Fig. 4). Siguieron las opciones de visitar lugares poco comunes, el respeto al medioambiente y el contacto con la comunidad local. Por último, el grupo conformado por las opciones de diversión y entretenimiento y otros, representaron una valoración significativamente menor a los otros factores.

Al evaluar el grado de satisfacción durante el viaje, la mayoría se refirió al "cuidado del medioambiente" y la información turística (Fig. 5). En segundo lugar estuvo la oferta turística en la región. El grupo con menor satisfacción incluyó a tres aspectos que no presentaron diferencias significativas entre sí: la conectividad dentro de la región, la conectividad con el resto del país y la diversión y el entretenimiento.

Respecto a las preferencias de destino en la Región de Magallanes, los turistas señalaron en primer lugar a Torres del Paine. En segundo lugar de las preferencias se ubicaron la colonia de elefantes marinos de Tierra del Fuego y el Cabo de Hornos. Estos dos destinos no presentaron diferencias significativas entre sí, pero tuvieron una preferencia significativamente mayor que los destinos urbanos tales como Puerto Williams, Puerto Natales y Punta Arenas (Fig. 6).

\section{Conservación del elefante marino del sur y sus hábitats en la $\mathrm{RBCH}$}

Respecto a la valoración del elefante marino, los turistas calificaron como de alta importancia de conservación y de visita al elefante marino dentro del circuito realizado. En una escala de 0 a 10 (de menor a mayor, respectivamente), la evaluación promedio fue de un 9,44 y 9,23, respectivamente (Fig. 7). En relación a la necesidad de conservar 
¿Cuáles de los siguientes aspectos en su viaje usted le da la mayor importancia?

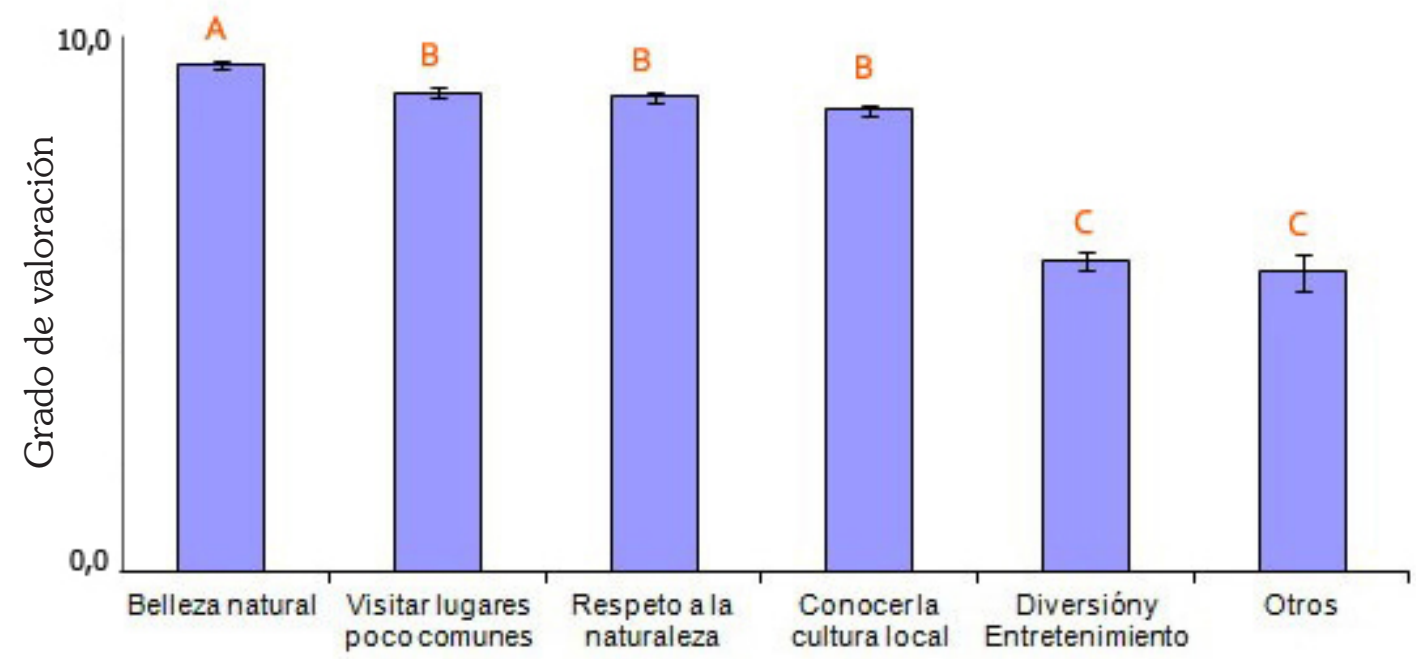

Fig. 4. Importancia de las motivaciones, en una escala de 0 a 10, que determinan el viaje de los turistas que visitan la $\mathrm{RBCH}$. Factores con las mismas letras no fueron significativamente diferentes entre sí.

Durante su estadía ¿Cuál fue el grado de satisfacción con relación a los siguientes servicios?

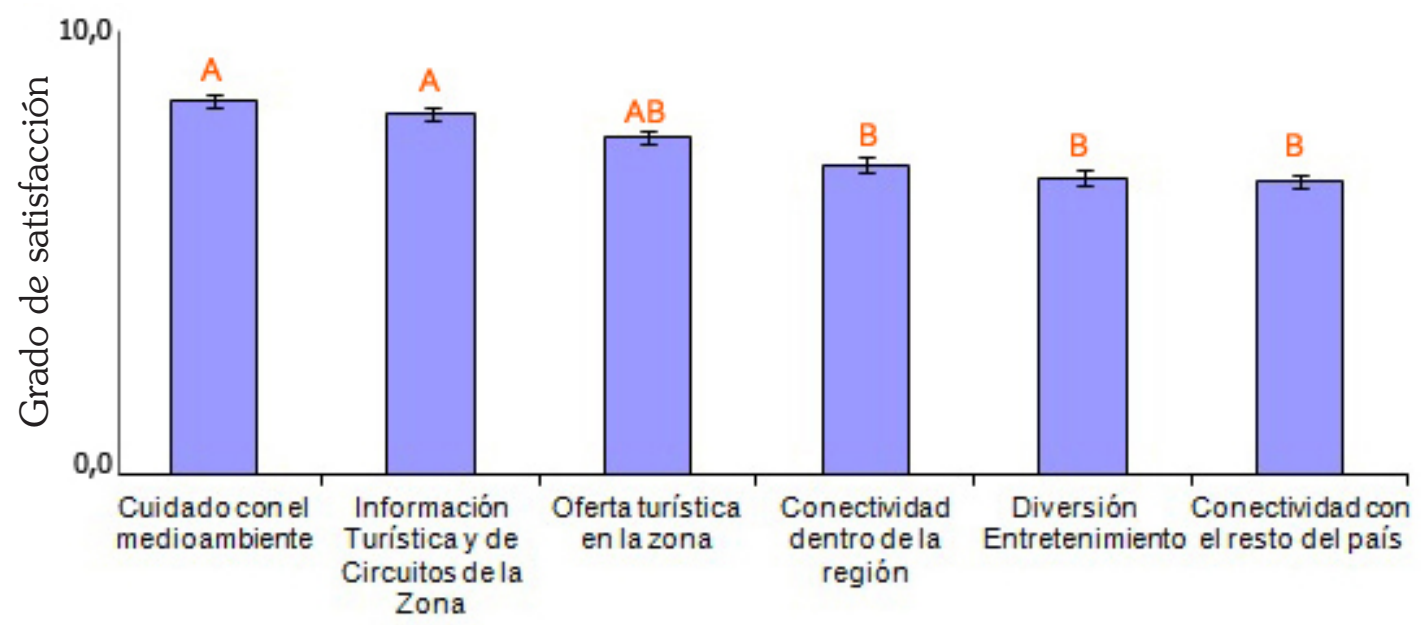

Fig, 5. Grado de satisfacción de las preferencias de los turistas a varios servicios durante la visita a la Región de Magallanes y Antártica Chilena. Servicios con las mismas letras no fueron significativamente diferentes entre sí.

esta especie, algunos señalaron la opción de crear áreas marinas protegidas, como por ejemplo bahía Ainsworth, que está zonificada como zona buffer o de amortiguamiento en la $\mathrm{RBCH}$, pero carece de una figura de protección legal (cfr. Rozzi et al. 2007). Los encuestados se manifestaron mayoritariamente a favor (64\%) de la creación de áreas marinas protegidas que protejan al elefante marino y sus hábitats (Fig. 8).

Colaboración con un

programa para la conservación

Al consultar a los turistas si les gustaría 
Orden de preferencia de lugares visitados

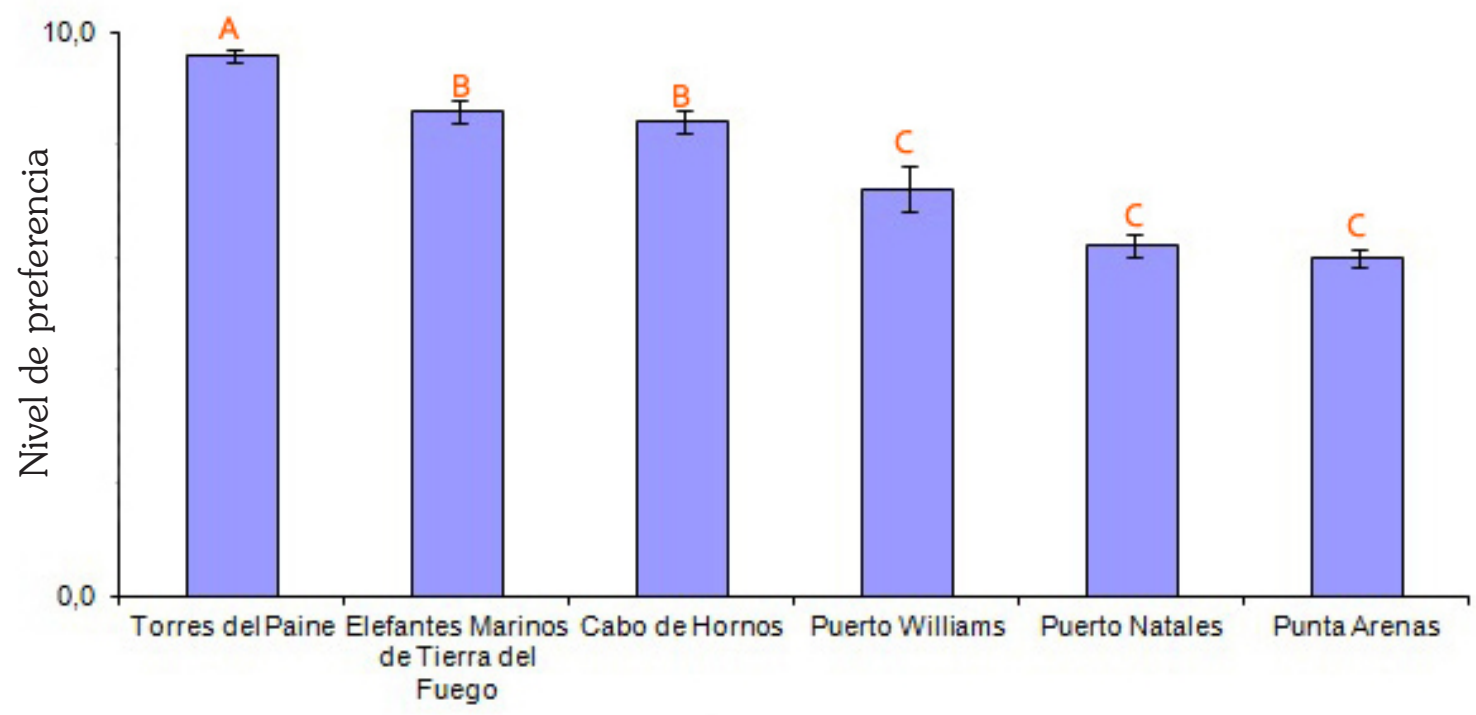

Fig. 6. Preferencias de los turistas por lugares dentro de la Región de Magallanes y Antártica Chilena. Variables con las mismas letras no fueron significativamente diferentes entre sí.

Valoraciones respecto al Elefante Marino

¿Cómo valora la importancia de conservar el Elefante Marino?

¿Qué importancia le asigna a la visita a los Elefantes Marinos dentro del circuito que usted está realizando?

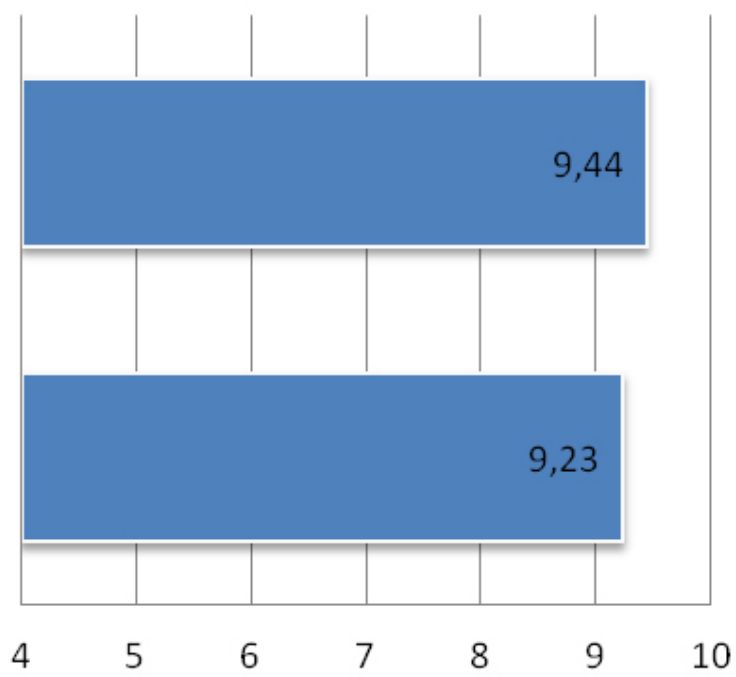

Fig. 7. Importancia que le asignan los turistas al elefante marino para la conservación y el circuito de visita.

colaborar para la protección del elefante marino, los encuestados se manifestaron mayoritariamente a favor de asumir un pequeño costo adicional en su circuito por la región que ayudara a financiar medidas de protección por parte del Estado de Chile para esta especie. Un $77,5 \%$ de los encuestados se pronunció a favor de esta medida, un $16,9 \%$ se manifestó en contra y un 5,6 no contestó la pregunta (Fig. 8). Análogamente, los encuestados respondieron afirmativamente respecto de colaborar para financiar medidas de conservación de los elefantes marinos y sus hábitats. Las respuestas afirmativas alcanzaron un $74,4 \%$, un $17,2 \%$ respondió negativamente esta pregunta, mientras que un $8,5 \%$ de los encuestados no la contestó (Fig. 9). 
Disposición a pagar para la conservación

A los encuestados que respondieron afirmativamente la pregunta anterior, se les consultó por el porcentaje del costo del pasaje del crucero que donarían a la conservación del elefante marino. Todos señalaron valores iguales o superiores al $0,5 \%$ del costo promedio de 2.000 dólares del pasaje, es decir, 10 dólares norteamericanos. Una mayoría $(53,5 \%)$ optó por las opciones de destinar un valor equivalente al $5 \%$ del pasaje $(34,7 \%)$ o al $10 \%$ del pasaje (18,8\%) (Figura 9). Es más del $50 \%$ de los visitantes está dispuesto a hacer un aporte de entre US\$100 o 200. Otro grupo significativo de pasajeros $(32 \%)$ optaría por donar un $1 \%$ del valor del pasaje, y un $7 \%$ de los pasajeros un $2 \%$ valor del pasaje; es decir, contribuir US $\$ 20$ o US\$40, respectivamente.

Al consultar al mismo grupo qué porcentaje del pasaje estarían dispuestos a destinar para colaborar para la conservación de la Patagonia, el $55,1 \%$ señaló valores iguales o superiores a US\$100; el 40,6\% estuvo dispuesto a pagar $5 \%$ del calor del pasaje, y $14,5 \%$ un aporte equivalente al $10 \%$ del pasaje (Fig. 9).
¿Piensa usted que las áreas marinas de protección pueden ser una buena alternativa para la Patagonia?

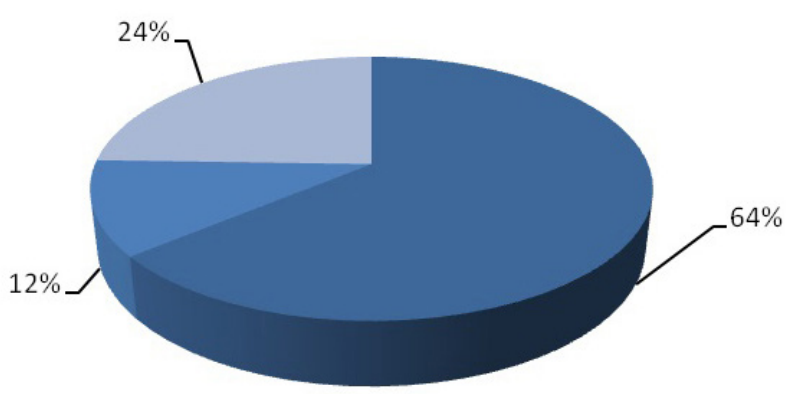

- Sí No No contesta / No sabe

Fig. 8. Disposición a colaborar con programas para la conservación de los elefantes marinos y de sus hábitats en la Patagonia y la $\mathrm{RBCH}$.

\section{DISCUSIÓN Y CONCLUSIONES}

\section{Perfil del turista}

El perfil del turista de Cruceros Australis coincide con el detectado para otros destinos

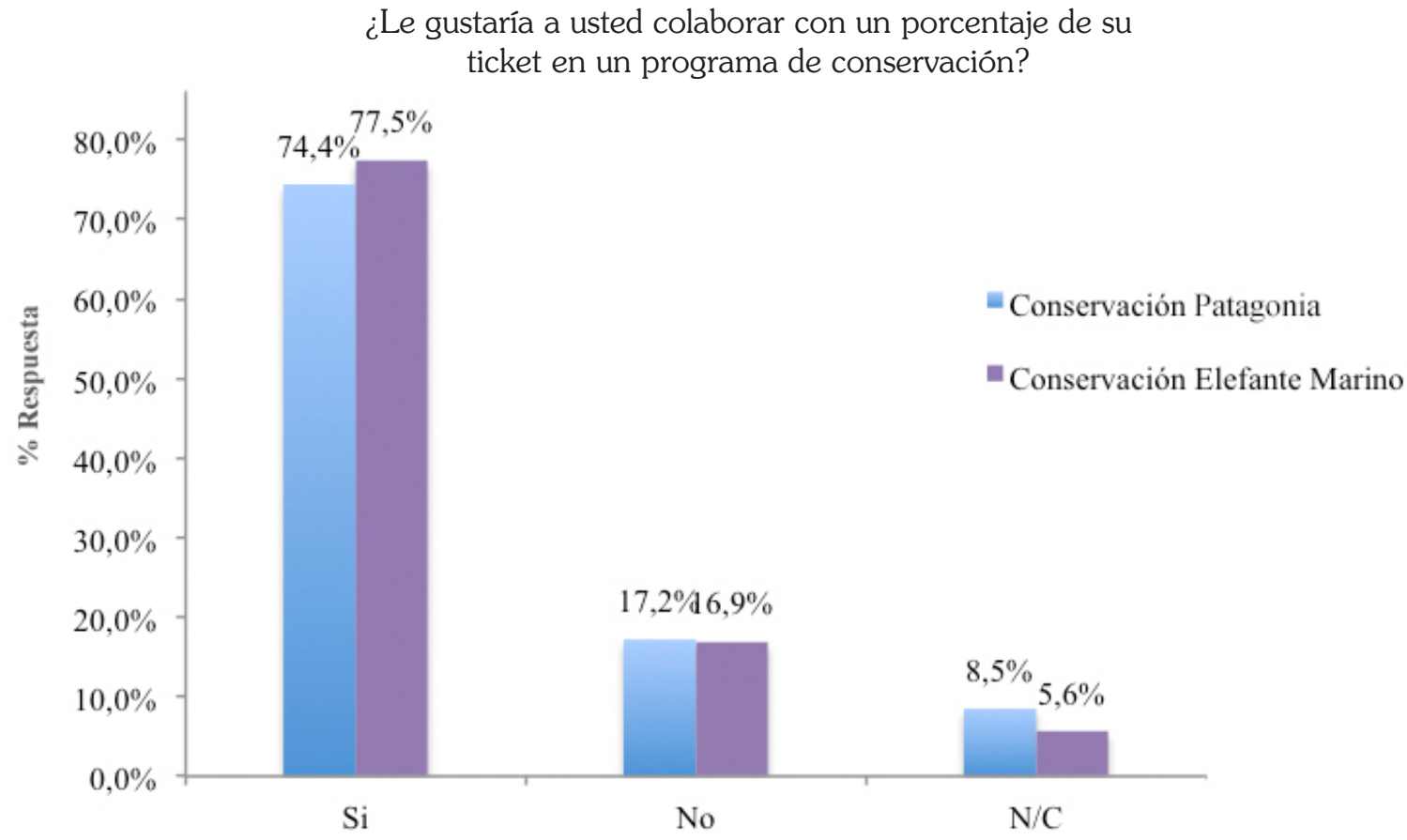

Fig. 9. Porcentaje del gasto del ticket en Cruceros Australis que los visitantes estarían dispuestos a donar para un programa protección de los elefantes marinos y de sus hábitats en la Patagonia y la $\mathrm{RBCH}$. 
de naturaleza. Por ejemplo, la proporción entre hombres y mujeres de $59 \%$ y $41 \%$ es muy similar a la encontrada en el estudio de perfiles de turistas en Ecuador que fue de $60 \%$ y $40 \%$, respectivamente (BCG, 2007). Este perfil educativo y etáreo también corresponde al rango típico de viajeros de larga distancia y con recursos para este tipo de viajes (BCG, 2007).

De acuerdo a los datos analizados, el cuidado del medioambiente fue uno de los factores con mayor valoración para los turistas en sus visitas a la $\mathrm{RBCH}$. Entre los aspectos más importantes estuvieron también la información turística y la oferta regional. El aspecto menos valorado fue la conectividad con el resto del país, lo que resulta coherente puesto que en la región no existe conectividad terrestre con el resto del país, razón por la cual los accesos se realizan preferentemente por vía aérea y fronteriza desde Argentina (BCG, 2007).

La región de Magallanes y Antártica Chilena incluye las mayores áreas protegidas del país, y el mayor porcentaje de territorio protegido de Chile (Armesto et al. 1998). Además, el archipiélago patagónico incluye más de $25.400 \mathrm{~km}$ de costa (Sielfeld, 1997). Por esta razón, no resulta extraño que los encuestados asignen la mayor importancia a la belleza natural y a la extensión de las áreas protegidas, dado que casi todos los circuitos turísticos de la región las incorporan como parte de la oferta.

De acuerdo a las características y preferencias de los turistas de Cruceros Austroalis, el segmento de mercado de turismo al cual pertenecen (sensu Drumm \& Moore, 2002) que mejor representa a los encuestados en esta investigación es el Turismo de Naturaleza. Más específicamente el ecoturismo, puesto que realizan actividades recreativas en contacto directo con la naturaleza y las expresiones culturales presentes en ella, con una actitud y compromiso de conocer, respetar, disfrutar y participar en la conservación de estos valiosos recursos (Drumm \& Moore, 2002; Vera et al. 1997; Zúñiga, 2007).

\section{Colaboración con un programa para la conservación}

La valoración que los turistas asignaron a la conservación de los elefantes marinos y a la biodiversidad de la Patagonia fue un resultado clave detectado en este estudio respecto de la satisfacción durante el recorrido, tanto en el circuito específico como en la región. Los turistas también fueron conscientes de la necesidad de contar con planes de conservación y áreas protegidas. Por estas razones, más del $97 \%$ de los turistas estuvo dispuesto a destinar un monto de entre 20 y 100 dólares estadounidenses para fines de conservación. Si se calcula la proporción que cada pasajero que se manifestó en favor de colaborar, y se aplican estos porcentajes al número de pasajeros de Cruceros Australis en el año 2012 (14.000 turistas), entonces se pueden estimar los montos que se podrían recaudar por cada segmento de turistas (Tabla 1). La sumatoria de montos parciales tiene un valor de aporte anual superior a 1.000.000 dólares estadounidenses (US\$1.190.700). Es importante notar que casi la mitad de este valor provendría de menos del $20 \%$

Tabla 1. Potencial aporte en US\$ para la conservación por 14 mil turistas que se embarcaron en Cruceros Australis en el año 2012

\begin{tabular}{ccccc}
\hline \% Ticket & \% Respuesta & $\begin{array}{c}\text { Valor aporte } \\
\text { individual }(\$ \text { US) }\end{array}$ & N $^{\circ}$ Turistas & $\begin{array}{c}\text { Valor aporte total } \\
\text { (\$ US) }\end{array}$ \\
\hline 0,5 & 2,3 & 10 & 322 & 3.220 \\
1 & 31,9 & 20 & 4.466 & 89.320 \\
2 & 7 & 40 & 980 & 39.200 \\
3 & 2,3 & 60 & 322 & 19.320 \\
3,5 & 2,8 & 70 & 392 & 27.440 \\
5 & 34,7 & 100 & 4.858 & 485.800 \\
10 & 18,8 & 200 & 2.632 & 526.400 \\
\hline TOTAL & & & 13.972 & 1.190 .700 \\
\hline
\end{tabular}


de los turistas del segmento dispuesto a pagar al menos US $\$ 200$ por persona por viaje. Más aun, el $53.5 \%$ de los turistas estuvo dispuesto a donar una base de US\$100 o 200 con un total de US $\$ 1.012 .200$, esto es el $85 \%$ de la recaudación total. Este hallazgo subraya la importancia de un turismo de alta calidad con turistas dispuestos a pagar 100 dólares o más por visitar un lugar singular a nivel mundial, como la Reserva de la Biosfera Cabo de Hornos (Rozzi et al. 2004, 2006a).

El nivel de aporte que están dispuestos a entregar los turistas encuestados es alto comparado con estudios realizados en otras zonas del país en lugares que son destinos de turismo masivo de tipo "sol y playa", donde la disposición a pagar no supera los US\$10 (Gelcich et al. 2013). El valor determinado para la $\mathrm{RBCH}$ está muy cercano a destinos tales como Galápagos, que cobra más de US\$100 por tratarse de un destino "único" (Higham \& Lück, 2007). Esto demuestra que hoy la Reserva de la Biosfera Cabo de Hornos representa un hito turístico a nivel mundial, con un valor comparable a la Reserva de la Biosfera Galápagos (García, 2004; Rozzi et al. 2010).

Se ha documentado que los turistas tienen una mayor disposición a colaborar con destinos exclusivos que conservan los hábitats y su vida silvestre. En contraste, en destinos que presentan degradación ambiental y grandes números de turistas la disposición a colaborar es mucho menor (Gelcich et al. 2013; Viteri \& Brandt, 2015). Un aumento del número de turistas a menudo implica una baja en el valor de conservación de la naturaleza y de los ingresos económicos por turismo, lo que ocurre cuando las visitas superan un número que va más allá del valor de coexistencia entre el turismo y la conservación (ver Fig. 2). Por esta razón, es fundamental determinar los efectos que tendrían las visitas no sólo en la población de los elefantes marinos en bahía Ainsworth, sino también en la pérdida del valor de conservación de todo el entorno. Es decir, el aumento de turistas en la $\mathrm{RBCH}$ podría implicar que cada turista esté dispuesto a colaborar menos en la conservación de las áreas protegidas y su vida silvestre, a pagar menos por las visitas turísticas, y por lo tanto, se obtendría un menor ingreso derivado de la actividad turista.

Por otra parte, en destinos similares a la Patagonia como Galápagos, uno de los grandes problemas para la conservación de la biodiversidad no sólo es el número de turistas, sino también la estructura de los circuitos que se realizan. Es decir, los turistas que viajan en tours cortos de un día están menos dispuestos a pagar para conservación que en aquellos circuitos de un mayor número de días. Se concluye que el mayor tiempo de la experiencia en un destino único se correlaciona positivamente con una mayor disposición a colaborar y conservar los sitios que se visitan (Viteri \& Brandt, 2015). Por lo tanto, los circuitos de corto tiempo llamados "fulldays" tienden a no ser una buena alternativa para el desarrollo de un turismo sustentable en destinos únicos como Galápagos, la $\mathrm{RBCH}$ y la Patagonia (Kirk, 2012).

Los turistas se manifestaron en favor no sólo de aportar a la conservación de los elefantes marinos, sino que también a la conservación de sus hábitats y la biodiversidad de la Patagonia Chilena. A partir de los resultados de este estudio, recomendamos ensayar un plan piloto para implementar este pago para la conservación que los turistas de cruceros están dispuestos a realizar en la $\mathrm{RCBH}$. Este pago podría generar un fondo de aproximadamente US\$2 millones/año considerando que el número de pasajeros de Cruceros Australis es de 21.000 pasajeros anuales a partir de la temporada 2015. Este tipo de turismo de intereses especiales podría ser implementando en otras áreas protegidas marinas y terrestres de la Región de Magallanes, que podría posicionarse como destinos únicos, tales como los parques nacionales Bernando O`Higgins y Kaweshkar. Podría motivar también la evaluación de la disposición a pagar por parte de turistas con intereses especiales que visitan áreas protegidas semi-degradadas, tales como la RB Torres del Paine.

\section{El elefante marino como especie carismática}

Junto a la belleza de los hábitats y paisajes, especies como el elefante marino son carismáticas puesto que sirven de símbolo y estimulan la conciencia pública hacia la importancia de la conservación de la biodiversidad (Arango et al. 2007). Estas especies también se conocen como especies bandera y pueden liderar campañas de conservación de la vida silvestre y aquellos hábitats que atraen a los turistas (Isasi-Catalá, 2011; Simberloff, 1998). Algunas especies, tales como el mono tití dorado en Brasil (Leontopithecus rosalia), el oso panda (Ailuropoda 
melanoleuca) y el carpintero negro (Campephilus magellanicus), han sido usadas de manera efectiva como imagen corporativa de campañas: el mono tití dorado para proteger el bosque atlántico de Brasil, el oso panda para conservar los bosques de bambú en China y como símbolo de la organización internacional WWF (World Wildlife Fund) y el carpintero negro para la protección de las aves más australes del planeta en la $\mathrm{RBCH}$ (Arango et al. 2007). Estas especies se han elegido también por su estado de conservación problemático Sus características tanto estéticas como su estado de conservación, permiten proponer al elefante marino del sur como una especie carismática para la conservación y el turismo sustentable en la $\mathrm{RBCH}$ y la región de Magallanes y Antártica Chilena. Esta investigación documenta, además, la importancia que esta especie tiene para los turistas que realizan el circuito en el Cruceros Australis en la $\mathrm{RBCH}$, quienes le asignan un valor muy alto respecto a la necesidad de su conservación (ver Fig. 7). Por lo tanto, esta nueva especie carismática podría inspirar campañas de conservación, no sólo para las poblaciones de su especie, sino que también para hábitats marinos y terrestres de la $\mathrm{RBCH}$.

\section{AGRADECIMIENTOS}

Los autores agradecen a Branco Ivelic por su valioso apoyo en la realización de las encuestas y a Enrique Poblete por el análisis estadístico de estas encuestas. Este artículo es resultado de la tesis de Magister en Ciencias de Christian Kirk en el programa de Conservación de Ambientes Subantárticos de la Universidad de Magallanes, Chile. Ricardo Rozzi agradece el apoyo del Instituto de Ecología y Biodiversidad (proyectos ICM P05 y CONICYT FB P23) y Apoyo a Centros Científicos y Tecnológicos de Excelencia con Financiamiento Basal CONICYT AFB170008.

\section{BIBLIOGRAFÍA}

Acevedo J., Aguayo-Lobo, A., Brito, J. L., Torres, D. Cáceres, B., Vila, A., Cardeña, M., \& Acuña, P. (2016). Review of the current distribution of southern elephant seals in the eastern South Pacific. New Zealand Journal of Marine and Freshwater Research, DOI: 10.1080/00288330.2015.1132746
Acuña, P., Aguayo-Lobo, A., Acevedo, J., Parra, G., \& Pastene, P. (2005). Origen de Mirounga leonina en seno Almirantazgo. Tierra del Fuego Chile. Symposium New Approaches to the Quaternary Sciences in Fuego Patagonia.

Aonken Consultores. (1982). Catastro y Evaluación de Recursos en Parque Nacional Hernando de Magallanes, Parque Nacional Alberto de Agostini y Reserva Forestal Holanda. Chile: Ediciones Ministerio de Agricultura, Corporación Nacional Forestal.

Arango, X., Rozzi, R., Massardo, F., Anderson, C., \& Ibarra, T. (2007). Descubrimiento e implementación del pájaro carpintero gigante (Campephilus magellanicus) como especie carismática: una aproximación biocultural para la conservación en la reserva de la biosfera de Cabo de Hornos. Magallania, 35, 71-88.

Armesto, J. J., Rozzi, R., Smith-Ramírez, C., \& Arroyo, M. T. K. (1998). Effective conservation targets in South American temperate forest. Science, 282, 1271-1272.

BCG - Boston Consulting Group (2007). Estudios de competitividad en clúster de la economía chilena sector turismo. Consejo de innovación. Gobierno de Chile. 202 pp.

Cáceres, B. (2013). Comportamiento reproductivo de la foca elefante (Mirounga leonina, Linneaus 1758) en bahía Ainsworth, Tierra del Fuego, Chile y los potenciales efectos del turismo. Tesis de grado para optar al título de biólogo marino. Universidad Austral de Chile.

CONAMA. (2002). Diagnóstico y Propuestas para la Conservación de la Biodiversidad en la XII Región. Gobierno de Chile.

Constantine, R. (1999). Effects of the tourism on marine mammals in New Zealand. Science for Conservation, 106, 1173-2946.

Drumm, A., \& Moore, A. (2002). An Introduction to Ecotourism Planning. Volume I. Ecotourism Development. A Manual for the Conservation Planners and Managers. Editing Singer, A publication for the capacity building. The Nature Conservancy.

García, M. (2004). Cape Horn Reserve. A challenge for ecotourism. En R. Rozzi, F. \& C. B.Massardo (Eds.), The Cape Horn Biosphere Reserve: a proposal of conservation and tourism to archive sustainable development at the southern end of the Americas (pp. 177-204). Anderson. Punta Arenas, Chile: Ediciones Universidad de Magallanes.

Gelcich, S., Amar, F., Valdebenito, A., Castilla, C., Fernández, M., Godoy, C., \& Biggs, D. (2013). Financing marine protected areas through visitor fees: Insights from 
tourist willingness to pay in Chile. Ambio, 42, 975984.

Gibbons, J. (2002). Bases para una estrategia para la conservación de mamíferos marinos en la XII Región. Informe Final. Comisión Nacional del Medio Ambiente, Región de Magallanes y Antártica Chilena, Chile. MS.

Gibbons, J., \& Miranda, C. (2001). Presencia del elefante marino del sur (Mirounga leonina) (Phocidae) en Seno Almirantazgo, Tierra del Fuego. Anales del Instituto de la Patagonia, Serie Ciencias Naturales, 29, 157-159.

Higginbottom, K. (2004). Wildlife Tourism; Impacts, Management and Planning. Cooperative research. Center for Sustainable Tourism. Australia.

Higham, J., \& Lück, M. (2007). Marine Wildlife and Tourism Management. Ucrania: Ed. Cabi.

Isasi-Catalá, E. (2011). Los conceptos de especies indicadoras, paraguas, bandera y claves: Su uso y abuso en la ecología de conservación. Interciencia, 36, 31-38.

Kirk, C. (2012). Estrategia Turística Sostenible en los Archipiélagos de la Patagonia Chilena. Tesis de Grado de Magister en Turismo Sostenible. Universidad de Cooperación Internacional de Costa Rica. .

Le Boeuf, B., \& Laws, R. M. (1994). Elephant Seals. Populations, Ecology, Behavior and Physiology. Berkeley, California: University of California Press.

Lewis, M. (1996). El Elefante Marino del Sur. Biología de la Especie, Descripción General de la Agrupación de la Península Valdés y Protocolos de Trabajo. Centro Nacional Patagónico. Fundación Patagónica Natural. Plan de Manejo Integral de la Zona Costera Patagónica. Informe Técnico $\mathrm{N}^{\circ}$ 6. MS.

Lewis, M., Campagna, C., Marin, M. R. \& Fernández, T. (2006). Southern elephant seal north of the Antarctic polar front. Antarctic Science, 18, 213-221.

MMA. (2013). Especies Según Estado de Conservación. Ministerio de Medioambiente (MMA). www. mma.gob.cl/.../doc/Unificada_Especie_Estado_ Conservacion_072013.xls (consultado 12 mayo 2016)

Rozzi, R., Massardo, F., \& Anderson, C. (2004). Reserva de Biosfera Cabo de Hornos. Una Perspectiva de la Conservación y Turismo para el Desarrollo Sustentable en el Extremo Austral de América Latina. Punta Arenas: Ediciones Universidad de Magallanes.

Rozzi, R., Massardo, F., Mancilla, A., Anderson, C., \& Plana, J. (2006a). Los Paisajes Virgenes de la Reserva de la Biosfera Cabo de Hornos. Punta Arenas: Ediciones Universidad de Magallanes.

Rozzi, R., Massardo, F., Anderson, C., Heidinger, K., \&
Silander Jr. J. (2006b). Ten principles for biocultural conservation at the southern tip of the Americas: the approach of the Omora Ethnobotanical Park. Ecology \& Society, 11(43), https://www.ecologyandsociety. org/vol11/iss1/art43/

Rozzi, R., Massardo, F., Mansilla, A., Anderson, C. B., Berghöfer, A., Mansilla, M., ... \& Russell, S. (2007). La Reserva de Biosfera Cabo de Hornos: un desafío para la conservación de la biodiversidad e implementación del desarrollo sustentable en el extremo austral de América. Anales del Instituto de la Patagonia, 35, 55-62.

Rozzi, R., Massardo, F., Cruz, F., Grenier, C., Muñoz, A., Mueller, E., \& Elbers, J. (2010). Galápagos and the Cape Horn: Ecotourism or greenwashing in two iconic Latin American archipelagoes? Environmental Philosophy, 7, 1-32.

Rozzi, R., Armesto, J. J., Gutiérrez, J. Massardo, F., Likens, G., Anderson, C. B., Poole, A.,... \& Kalin, M. T. (2012). Integrating ecology and environmental ethics: Earth stewardship in the southern end of the Americas. BioScience, 62, 226-236.

SERNATUR. (2004). Informe Final. Cuenta Satélite de Turismo XII Región. Punta Arenas, Chile: Gobierno Regional de Magallanes y Antártica Chilena.

Sielfeld, W. (1997). Las áreas protegidas de la XII Región de Chile en la perspectiva de los mamíferos marinos. Estudios Oceanológicos, 16, 87-107.

Simberloff, D. (1998). Flagships, umbrellas, and keystones: is a single species management passé in the landscapes era? Biological Conservation, 83, 247-257.

Sokal, R. R., \& Rohlf, F. J. (2012). Biometry: The Principles and Practice of Statistics in Biological Research. $4^{\text {th }}$ Edition. New York: W. H. Freeman.

Sustentable. (2006). Informe Final Proyecto "Parque Regional Tierra del Fuego". Ministerio de Bienes Nacionales. Santiago de Chile.

UICN (2014). Perspectiva del Patrimonio Mundial de la UICN 2014. Evaluacion sobre la conservacion de todos los sitios naturales del Patrimonio Mundial.

Vera, J. F., Palomeque, F., Marchena, M., \& Salvado, A. (1997). Análisis Territorial del Turismo. Una Nueva Geografía del Turismo. Barcelona: Editorial Ariel.

Viteri, C., \& Brandt, S. (2015). Managing tourism in the Galapagos Islands through price incentives: A choice experiment approach. Ecological Economics, 117, 1-11.

Zúñiga, R. (2007). Plan de Desarrollo Turístico para la Hacienda San Fernando. Cantón San Miguel de los Bancos, Provincia de Pichincha. Ecuador: Universidad Tecnológica Equinoccial. 